\title{
Macular configuration determined by optical coherence tomography after idiopathic macular hole surgery with or without internal limiting membrane peeling
}

\author{
R Uemoto, S Yamamoto, T Aoki, I Tsukahara, T Yamamoto, S Takeuchi
}

Br J Ophthalmol 2002;86:1240-1242

\begin{abstract}
Aims: To evaluate the effect of pars plana vitrectomy (PPV) with or without internal limiting membrane (ILM) peeling on the closure and configuration of idiopathic macular holes (IMH).

Methods: PPV was performed for IMH on 44 eyes with ILM peeling (ILM peeled group) and on 42 eyes without ILM removal (ILM preserved group). Optical coherence tomography (OCT) was performed on 34 ILM peeled eyes and 14 ILM preserved eyes after successful surgery. The repaired macular holes were classified by the OCT images as being of "good shape" (nearly normal foveal contour) or "poor shape" (abnormal foveal contour with flat fovea and steep edge, or with a thick retina without a foveal pit). Results: The anatomical closure rate was significantly higher in the ILM peeled group (93.2\%) than in the ILM preserved group $(76.2 \%)(p=0.028)$. In the ILM peeled group, 31 eyes had a fovea of good shape and three eyes had a fovea with a poor shape, while in the ILM preserved group, six eyes had a fovea of good shape and eight eyes had a fovea of poor shape. The percentage of eyes with good macular configuration in the ILM peeled group was significantly higher than in the ILM preserved group ( $p=$ $0.0003)$. No significant difference was found in the postoperative visual acuity and the increase of visual acuity between the ILM peeled group and the ILM preserved group ( $p=0.26$, and $p=0.91$ respectively). There was also no significant difference in the postoperative visual acuity and improvement in visual acuity between eyes with a fovea of good shape and those with fovea of poor shape fovea ( $p=0.99$ and $p=0.66$, respectively).

Conclusions: ILM peeling may provide better anatomical success and recovery of the macular shape, but the postoperative visual acuity and improvement of visual acuity were not related to the morphological results.
\end{abstract}

t has been reported that idiopathic macular holes (IMHs) may be formed from the tangential traction of the acellular prefoveal vitreous and possibly by a contraction of the cellular constituents in the prefoveal vitreous. ${ }^{1}$ Earlier investigators suggested that the macular hole may enlarge by the contractile forces generated by glial cells that migrate onto the inner surface of the internal limiting membrane (ILM). ${ }^{1}$ Since then, the removal of the ILM during macular hole surgery has been widely advocated and excellent anatomical success rates have been reported..$^{2-}$

The ILM is the basal lamina of the Müller cells, and its removal should alter the morphology of the Müller cells. However, the effects of ILM removal on the macular morphology has not determined as yet. We have reviewed the cases of IMH that underwent pars plana vitrectomy (PPV) with and without ILM peeling in terms of the anatomical closure rates and the

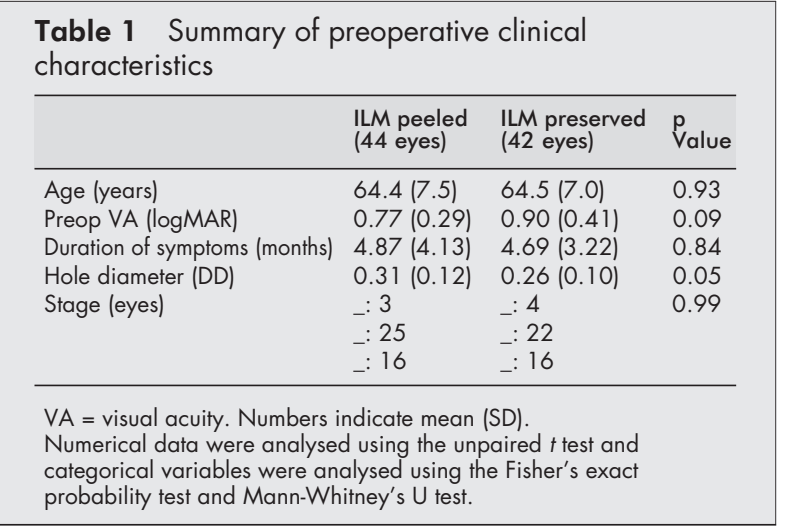

improvement of visual acuity. We also evaluated the configuration of the repaired macular holes after PPV by optical coherence tomography (OCT).

\section{PATIENTS AND METHODS}

We reviewed the charts of 86 consecutive eyes of 86 patients who had undergone PPV for IMH from June 1996 through April 2001 at the Sakura Hospital of Toho University. The postoperative follow up period ranged from 6 to 65.7 (mean 22.4) months. PPV combined with ILM peeling was performed on 44 eyes (ILM peeled group) and without ILM peeling on 42 eyes (ILM preserved group). This study was a sequential one, because the ILM peeling has been performed in almost all macular hole surgeries since August 1999. Baseline demographics for the patients in the two groups are given in Table 1.

The following characteristics were analysed; age, sex, estimated duration of visual symptoms, stage and size of macular holes, anatomical outcomes, preoperative and postoperative best corrected visual acuity converted to log MAR units, lens status, tamponade agent, the intraoperative and postoperative complications, and preoperative and postoperative OCT images. An anatomical success - that is, closure, was defined by ophthalmoscopic and OCT examinations as the complete disappearance of the hole and the flattening of the entire circumference of the macular hole against the retinal pigment epithelium. This end point corresponds with the definition of the "flat/closed" status described by Tornambe et al. ${ }^{6}$

OCT was performed postoperatively on 48 successfully treated eyes using the Humphrey model 2000 (Humphrey Instruments, San Leandro, CA, USA). There were 34 ILM peeled eyes and 14 ILM preserved eyes. The OCT examinations have been performed in all cases consecutively since September 1998 when the instrument was available in our institution. 


\begin{tabular}{|c|c|c|c|}
\hline & $\begin{array}{l}\text { ILM peeled } \\
\text { (44 eyes) }\end{array}$ & $\begin{array}{l}\text { ILM preserved } \\
\text { (42 eyes) }\end{array}$ & $\mathrm{p}$ Value \\
\hline Anatomical closure (eyes) & 41 of 44 & 32 of 42 & 0.028 \\
\hline $\begin{array}{l}\text { Postop VA (logMAR) } \\
\text { All cases } \\
\text { Initial success cases }\end{array}$ & $\begin{array}{l}0.42(0.39) \\
0.38(0.37)\end{array}$ & $\begin{array}{l}0.53(0.48) \\
0.33(0.33)\end{array}$ & $\begin{array}{l}0.26 \\
0.60\end{array}$ \\
\hline $\begin{array}{l}\text { Improved VA (logMAR) } \\
\text { All cases } \\
\text { Initial success cases }\end{array}$ & $\begin{array}{l}0.35(0.41) \\
0.38(0.41)\end{array}$ & $\begin{array}{l}0.37(0.52) \\
0.55(0.43)\end{array}$ & $\begin{array}{l}0.83 \\
0.08\end{array}$ \\
\hline $\begin{array}{l}\text { Lens status (postop) } \\
\text { Pseudophakia/phakia }\end{array}$ & $32 / 11$ & $38 / 4$ & 0.11 \\
\hline $\begin{array}{l}\text { Tamponade agent } \\
\mathrm{C}_{3} \mathrm{~F}_{8} / \mathrm{SF}_{6} / \text { room air }\end{array}$ & $3 / 30 / 10$ & $28 / 12 / 2$ & $<0.0001$ \\
\hline $\begin{array}{l}\text { OCT image (eyes) } \\
\text { Good shape }\end{array}$ & 31 of 34 & 6 of 14 & 0.00029 \\
\hline $\begin{array}{l}\text { Intraoperative complication } \\
\text { Retinal breaks } \\
\text { Retinal haemorrhage }\end{array}$ & $\begin{array}{l}\text { ns (eyes) } \\
3 \\
2\end{array}$ & $\begin{array}{l}4 \\
4\end{array}$ & $\begin{array}{l}0.65 \\
0.37\end{array}$ \\
\hline $\begin{array}{l}\text { Postoperative complication } \\
\text { ERM }\end{array}$ & $\begin{array}{l}\text { s (eyes) } \\
3\end{array}$ & 1 & 0.33 \\
\hline
\end{tabular}

$\mathrm{VA}=$ visual acuity, $E R M=$ epiretinal membrane. Numbers indicate mean $(S D)$

Numerical data were analysed using the unpaired $t$ test, and categorical variables were analysed using the Fisher's exact probability test and $\chi^{2}$ test for independence with Yates's correction.

The patient's pupil was fully dilated with topical $0.5 \%$ tropicamide, and the fundus was scanned with a probe beam positioned so that the horizontal and vertical scans crossed the central fovea as determined from fundus photographs. The scan length was usually $2.8 \mathrm{~mm}$. We repeated the OCT examinations several times and used the better images for the measurements.

The macular hole surgery included PPV accompanied by ILM peeling with or without indocyanine green (ICG) staining, ${ }^{78}$ fluid-air exchange, and injection of room air or $20 \%$ sulphur hexafloride $\left(\mathrm{SF}_{6}\right)$ gas, or $14 \%$ perfluoropropane $\left(\mathrm{C}_{3} \mathrm{~F}_{8}\right)$ gas. The patients were instructed to maintain a prone position for about 1 week postoperatively. Simultaneous phacoemulsification and aspiration for cataracts with intraocular lens implantation was performed on 26 ILM peeled eyes and 36 ILM preserved eyes. No significant difference was observed in the lens status between two groups.

The numerical data were analysed using the unpaired $t$ test, and the categorical variables were analysed using MannWhitney's U test and the Fisher's exact probability test. A p value of 0.05 or less was considered statistically significant.

\section{RESULTS}

There was no significant difference in the ages, preoperative visual acuity, estimated duration of visual symptoms, macular hole diameter, and the stage of hole between the ILM peeled eyes and the ILM preserved eyes (Table 1).

A comparison of the surgical results between the two groups is shown in Table 2 . The anatomical success rate was significantly higher in the ILM peeled eyes $(p=0.028)$, but the postoperative visual acuity, the increase of visual acuity, and the postoperative lens status was not correlated with the ILM peeling ( $\mathrm{p}=0.26, \mathrm{p}=0.83$, and $\mathrm{p}=0.11$, respectively). A significantly smaller number of the ILM peeled eyes had the $\mathrm{C}_{3} \mathrm{~F}_{8}$ tamponade $(\mathrm{p}<0.0001)$, because the ILM peeling was apparently promising for the macular hole closure. When the improvement in visual acuity was analysed only for eyes with successful closure at the initial surgery, the improvement was slightly higher in the ILM preserved eyes; however, the difference was not statistically significant $(\mathrm{p}=0.08)$.

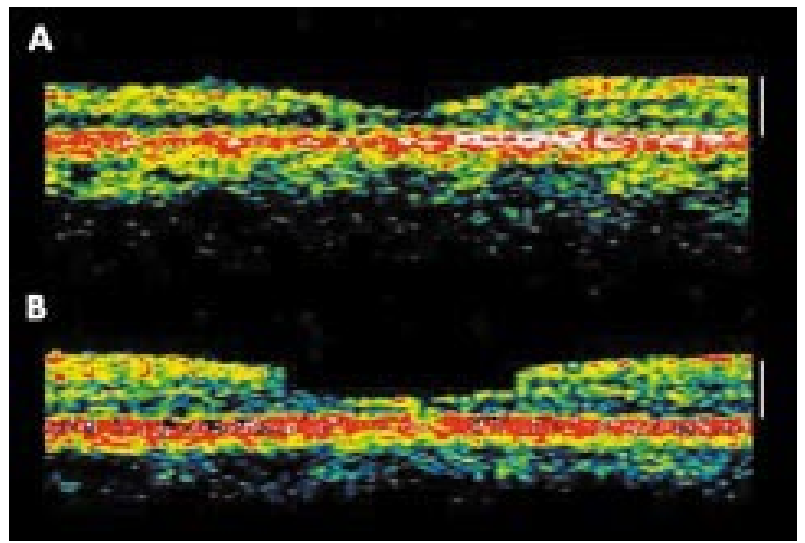

Figure 1 (A) OCT image obtained from a horizontal scan of a 68 year old patient after successful macular hole surgery. The foveal shape was classified as a "good shape." The OCT image shows a highly reflective band corresponding to the retinal pigment epithelium, and the choriocapillaris is covered by a layer that shows mild to moderate backscattering with a smooth circular and concave surface similar to that of the normal fovea. (B) Horizontal OCT scan of a 65 year old patient after successful macular hole surgery. The foveal shape was classified as a "poor shape." This OCT image shows the presence of a highly reflective band surrounding the macula regions. The macula edge is sharp and the macula is flatly sunken. The calibration markers indicate $250 \mu \mathrm{m}$.

The foveas of the initially repaired macular holes were classified as being in "good shape" when they showed nearly normal foveal contour (Fig 1A), or in "poor shape" when they showed abnormal foveal contour with a flat fovea with steep edges, or with a thick retina and absent foveal pit (Fig 1B) from the OCT images. This classification was performed by investigators who were blinded to clinical findings, surgical technique, and visual acuity at the definition. Among 34 ILM peeled eyes, 31 eyes had a fovea with a "good shape" and three eyes had a fovea with a "poor shape," whereas among the 14 ILM preserved eyes, six eyes had a fovea with a "good shape" and eight eyes had a fovea with a "poor shape." This difference in the distribution of the postoperative foveal shape was significant $(\mathrm{p}=0.00029)$.

There was no significant difference in the postoperative visual acuity and the improvement of visual acuity in the eyes with a fovea of "good shape" and those with a fovea of "poor shape" in the ILM peeled group ( $\mathrm{p}=0.99$ and $\mathrm{p}=0.66$, respectively).

The postoperative complications included epiretinal membranes in three ILM peeled eyes and one ILM preserved eye.

\section{DISCUSSION}

ILM peeling has recently become a common procedure during macular hole surgery because this procedure has been reported to lead to higher anatomical success rates. ${ }^{2-5}$ However, the effect of the ILM peeling on the retinal morphology and visual functions has not yet been presented in detail.

The Müller cell cone, which is an inverted cone-shaped zone of specialised Müller cells that form the base of the fovea, serves as a plug to bind together the photoreceptor cells in the foveola and supports the foveola structurally. ${ }^{10}$ Therefore, ILM peeling may lead to the loss of structural supports of the fovea. In addition, ILM peeling may also completely release the tangential traction of the residual prefoveal vitreous after the posterior vitreous detachment or the contraction of the epiretinal cellular constituents adjacent to the macular hole. These two factors should then alter the mobility of the macular hole edge. Furthermore, the contraction of glial cells that proliferated following the macular hole surgery with ILM peeling would lead to a centripetal movement of the photoreceptors. ${ }^{11}$ Subsequently, the mobility of the edges of 
the hole and glial proliferation would lead to improvement of the macula shape and a higher anatomical success rate as found in our study. There have been two clinicopathological studies reporting that eyes had ILM peeling showed anatomicalal repair of macular holes with the smallest defects. ${ }^{11}{ }^{12}$

Our results showed that the postoperative visual acuity was not correlated with the macular configuration as evaluated by OCT postoperatively. Thus, the improvement in the foveal shape is not correlated with the visual recovery. This result confirms findings reported by Brooks that vision was similar, as long as the hole was closed, irrespective of the ILM peeling. ${ }^{3}$ The reason for this lack of correlation is that OCT can evaluate only the morphology of the macula areas and does not provide functional information on the retinal pigment epithelium, the photoreceptors, and Müller cells of the sensory retina.

Moreover, the visual results were not correlated with the ILM peeling, even in cases with anatomical success, although the closure rate was significantly improved in the ILM peeled group. In general, the higher anatomical success rate should lead to better visual recovery. The Müller cell footplates that make up the outer portions of the ILM must sustain some degree of injury by the peeling. Therefore, even though the ILM peeling may enhance anatomical success, it may be an unfavourable factor for visual recovery because of damage to the inner retinal tissues such as the Müller cell and the possibility of the disturbed structure in the fovea. In support of this, focal macular electroretinography has shown a selective delay of b-wave recovery after macular hole surgery with the ILM peeling. ${ }^{13}$

It has been reported that complete ILM peeling leads to higher anatomical and visual success, while unsuccessful ILM peeling may induce a stronger stimulation of gliosis and lead to the higher anatomical success rate at the expense of a lower visual success because of damage to adjacent inner retinal elements. ${ }^{14}$

In this study, postoperative OCT results were simply divided into "good shape" or "poor shape" by masked observers, because objective grading of the foveal configuration currently appeared difficult. Objective criteria to grade the OCT findings would be desirable and evaluate surgical results more quantitatively.

The present clinical study demonstrated that ILM peeling leads to the recovery the foveal morphological changes and promotes anatomical success; however, there was no relation between visual improvement and morphological results. Further study will be needed to clarify potential influence of this surgical procedure on the retina in terms of the function and histopathology.

\section{Authors' affiliations}

R Uemoto, S Yamamoto, T Aoki, I Tsukahara, T Yamamoto,

S Takeuchi, Department of Ophthalmology, Toho University Sakura Hospital, Sakura, Japan

Correspondence to: Shuichi Yamamoto, MD, Department of Ophthalmology, Toho University Sakura Hospital, 564-1 Shimoshizu, Sakura, Chiba 285-8741, Japan; shuyama@med.toho-u.ac.jp

Proprietary interest: None of the authors or their family members has any proprietary or financial interest in the instruments used in this study.

Accepted for publication 19 June 2002

\section{REFERENCES}

1 Yoon H, Brooks HL Capone A Jr, et al. Ultrastructural features of tissue removed during idiopathic macular hole surgery. Am J Ophthalmol 1996; 122:67-75.

2 Park DW, Sipperley JO, Sneed SR, et al. Macular hole surgery with internal-limiting membrane peeling and intravitreous air. Ophthalmology 1999;106:1392-7 (discussion 1397-8).

3 Brooks HL. Macular hole surgery with and without internal limiting membrane peeling. Ophthalmology 2000;107:1939-49.

4 Mester V, Kuhn F. Internal limiting membrane removal in the management of the full-thickness macular holes. Am J Ophthalmol 2000;129:769-77.

5 Kwok AK, Li WW, Pang CP, et al. Indocyanine green staining and removal of internal limiting membrane in macula hole surgery: histology and outcome. Am J Ophthalmol 2001;132:178-83.

6 Tornambe PE, Poliner LS, Cohen RG, et al. Definition of macular hole surgery end points: elevated/open, flat/open, flat/closed. Retina 1998;18:286-7.

7 Kadonosono K, Itoh N, Uchio E, et al. Staining of internal limiting membrane in macular hole surgery. Arch Ophthalmol 2000;118:1116-

8 Da Mata AP, Burk SE, Riemann CD, et al. Indocyanine green-assisted peeling of the retinal internal limiting membrane during vitrectomy surgery for macula hole repair. Am J Ophthalmol 2001;108:1 187-92.

9 Yamada E. Some structural features of the fovea centralis in the human retina. Arch Ophthalmol 1969;82:151-9.

10 Gass JDM. Muller cell cone, an overlooked part of the anatomy of the fovea centralis. Arch Ophthalmol 1999;117:821-3.

11 Funata M, Wendel RT, de la Cruz Z, et al. Clinicopathologic study of bilateral macular holes treated with pars plana vitrectomy and gas tamponade. Retina 1992; 12:289-98.

12 Madreperla SA, Geiger GL, Funata M, et al. Clinicopathologic correlation of a macular hole treated by cortical vitreous peeling and gas tamponade. Ophthalmology 1994:101:682-6.

13 Terasaki H, Miyake Y, Nomura R, et al. Focal macular ERGs in eyes after removal of macular ILM during macular hole surgery. Invest
a Ophthalmol Vis Sci 2001;42:229-34

14 Smiddy W, Fever W, Cordahi G. Internal limiting membrane peeling in macular hole surgery. Ophthalmology 2001;108:1471-8. 